QUARTERLY OF APPLIED MATHEMATICS

VOLUME LXIX, NUMBER 3

SEPTEMBER 2011, PAGES 509-528

S $0033-569 X(2011) 01218-0$

Article electronically published on May 6, 2011

\title{
GLOBAL SMOOTH SOLUTIONS FOR THE COMPRESSIBLE VISCOUS AND HEAT-CONDUCTIVE GAS
}

\author{
BY \\ YUMING QIN (Department of Applied Mathematics, Donghua University, Shanghai 201620, \\ People's Republic of China), \\ GUILI HU (College of Sciences, Donghua University, Shanghai 201620, People's Republic of China), \\ AND \\ TAIGE WANG (College of Sciences, Donghua University, Shanghai 201620, People's Republic of \\ China)
}

Abstract. This paper is concerned with the global existence of smooth solutions to a system of equations describing one-dimensional motion of a self-gravitating, radiative and chemically reactive gas. We have proved that for any arbitrary large smooth initial data, the problem under consideration admits a unique globally smooth (classical) solution. Our results have improved those results by Umehara and Tani ([J. Differential Equations, 234(2007), 439-463; Proc. Japan Acad., 84, Ser. A(2008), 123-128]) and also by Qin, $\mathrm{Hu}$, Huang, and Ma.

1. Introduction. Radiation hydrodynamics [17, [19, 30] describes the propagation of thermal radiation through a fluid or gas. Similarly to ordinary fluid mechanics, the equations of motion are derived from conservation laws for macroscopic quantities. However, when radiation is present, the classical "material" flow has to be coupled with the radiation, which is an assembly of photons and needs a priori a relativistic treatment (the photons are massless particles traveling at the speed of light). The whole problem under consideration when the matter is in local thermodynamical equilibrium (LTE) is thus a coupling between standard hydrodynamics for the matter and a radiative transfer equation for the photon's distribution; through a suitable description, as in plasma when the radiation is LTE with matter and velocities are not too large, a nonrelativistic one-temperature description is possible [17], 30]. Moreover, if the matter is extremely

Received January 4, 2010.

2000 Mathematics Subject Classification. Primary 35B41.

Key words and phrases. Global solution; free-boundary problem; self-gravitation; radiative gas; reactive gas; Lagrangian mass coordinate.

The authors were supported in part by the NNSF Grant of China \#10871040.

E-mail address: yuming-qin@hotmail.com

E-mail address: hgl-8507@163.com

E-mail address: tigerwtg@hotmail.com 
radiative opaque, so that the matter-free path of photons is much smaller than the typical length of the flow, we obtain a simplified description (radiation hydrodynamics in the diffusion limit) which amounts to solving a standard hydrodynamical (compressible Navier-Stokes) problem system with additional correction terms in the pressure, the internal energy and the thermal conduction. To describe richer physical processes, for simplicity we may consider the fluid as reactive and couple the dynamics with the firstorder chemical kinetics of combustion type, namely the one-order Arrhenius kinetics. Although it is simplified, this model can be proved to model correctly some astrophysical situations of interest such as stellar evolution or interstellar medium dynamics (see, e.g., [4]).

In this paper, we are concerned with the free-boundary problem describing the motion of a compressible, viscous and heat-conducting gas which is self-gravitating, radiative and chemically reactive. Such a gaseous motion, especially in the processes of the unimolecular reactions whose kinetic order is one, is described by the following equations in Lagrangian mass coordinates:

$$
\begin{aligned}
& v_{t}=u_{x} \\
& u_{t}=\left(-p+\mu \frac{u_{x}}{v}\right)_{x}-G\left(x-\frac{1}{2}\right), \\
& e_{t}=\left(-p+\mu \frac{u_{x}}{v}\right) u_{x}+\left(\kappa \frac{\theta_{x}}{v}\right)_{x}+\lambda \phi z, \\
& z_{t}=d\left(\frac{z_{x}}{v^{2}}\right)_{x}-\phi z
\end{aligned}
$$

in $\Omega \times(0, \infty)$ with $\Omega=(0,1)$. Here the specific volume $v=v(x, t)$, the velocity $u=u(x, t)$, the absolute temperature $\theta=\theta(x, t)$ and the mass fraction of the reactant $z=z(x, t)$ are the unknown quantities, and the positive constants $\mu, G, d$ and $\lambda$ are the bulk viscosity, the Newtonian gravitational constant, the species diffusion coefficient and the difference in heat between the reactant and the product, respectively. The pressure $p$ and the internal energy per unit mass $e$ are defined by

$$
p=p(v, \theta)=\frac{R \theta}{v}+\frac{a}{3} \theta^{4}, \quad e=e(v, \theta)=c_{V} \theta+a v \theta^{4},
$$

where the positive constants $R, c_{V}$ and $a$ are the perfect gas constant, the specific heat capacity at constant volume and the radiation-density constant, respectively. The second terms on the right-hand side of both relations in (1.5) stand for the effect of the radiation phenomena, whose forms are given by the famous Stefan-Boltzmann law. In the radiating regime, we naturally take into account the heat flux from the radiative contribution, not only from the heat-conductive contribution. As such a simple one (see [5], 27], 28, [21]), we assume that the thermal conductivity $\kappa=\kappa(v, \theta)$ takes the form

$$
\kappa(v, \theta)=\kappa_{1}+\kappa_{2} v \theta^{q}
$$

with positive constants $\kappa_{1}, \kappa_{2}$ and $q$. Furthermore we assume that the reaction rate function $\phi=\phi(\theta)$ is defined, from the Arrhenius law, by

$$
\phi(\theta)=K \theta^{\beta} e^{-A / \theta},
$$


where the positive constants $K$ and $A$ are the coefficient of rate of the reactant and the activation energy, respectively, and $\beta$ is a nonnegative real number.

We consider (1.1)-(1.4) subject to the following boundary condition:

$$
\left.\left(\sigma, \theta_{x}, z_{x}\right)\right|_{x=0,1}=\left(-p_{e}, 0,0\right), \quad t>0,
$$

with the stress $\sigma=-p+\mu \frac{u_{x}}{v}$ and the external pressure $p_{e}$ (a positive constant), and the initial condition

$$
\left.(v, u, \theta, z)\right|_{t=0}=\left(v_{0}(x), u_{0}(x), \theta_{0}(x), z_{0}(x)\right), \quad x \in[0,1] .
$$

Without loss of generality, we may assume that (see, e.g., [27], [28], [21])

$$
\int_{0}^{1} u_{0}(x) d x=0 .
$$

The notation in this paper will be as follows: Let $m$ be a nonnegative integer and $0<\alpha, \alpha^{\prime}<1$. By $C^{m+\alpha}(\Omega)$ we denote the spaces of functions $u=u(x)$ which have bounded derivatives up to order $m$ and $d^{m} u / d x^{m}$ is uniformly Hölder continuous with exponent $\alpha$. Let $T$ be a positive constant and $Q_{T}=\Omega \times(0, T)$. For a function $u$ defined on $Q_{T}$, we say that $u \in C_{x, t}^{\alpha, \alpha^{\prime}}\left(Q_{T}\right)$ if

$$
|u|^{(0)}=\sup _{(x, t) \in Q_{T}}|u(x, t)|<\infty
$$

and $u$ is uniformly Hölder continuous in $x$ and $t$ with exponents $\alpha$ and $\alpha^{\prime}$, respectively. Its norm is denoted by $|\cdot|_{\alpha, \alpha^{\prime}}$. We also say that $u \in C_{x, t}^{2+\alpha, 1+\alpha / 2}\left(Q_{T}\right)$ if $u$ is bounded, has bounded derivative $u_{x}$, and $\left(u_{x x}, u_{t}\right) \in\left(C_{x, t}^{\alpha, \alpha / 2}\left(Q_{T}\right)\right)^{2}$. Its norm is denoted by $|\cdot|_{2+\alpha, 1+\alpha / 2} \cdot L^{p}, 1 \leq p \leq+\infty, W^{m, p}, m \in N, H^{1}=W^{1,2}, H_{0}^{1}=W_{0}^{1,2}$ denote the usual (Sobolev) spaces on $[0,1]$. In addition, $\|\cdot\|_{B}$ denotes the norm in the space $B$; we also put $\|\cdot\|=\|\cdot\|_{L^{2}(0,1)}$. We use $C_{0}$ to denote the generic positive constant depending on the initial data, but independent of $t$. The constant $C>0$ stands for the generic positive constant depending only on the initial data and $T>0$. Without danger of confusion, we will use the same symbol to denote the state functions as well as their values along a dynamic process, e.g., $p(v, \theta), p(v(x, t), \theta(x, t))$ and $p(x, t)$.

Let $E=E_{1} \cup E_{2}$ be a set in the $(q, \beta)$-plane in $R^{2}$, where

$$
\begin{aligned}
& E_{1}=\left\{(q, \beta) \in R^{2}: 2 \leq q<3,0 \leq \beta<2 q+6\right\}, \\
& E_{2}=\left\{(q, \beta) \in R^{2}: 3 \leq q, \quad 0 \leq \beta<q+9\right\} .
\end{aligned}
$$

Our results read as follows:

Theorem 1.1. Let $(q, \beta) \in E$ and $\alpha \in(0,1)$. Assume that the initial data

$$
\left(v_{0}, u_{0}, \theta_{0}, z_{0}\right) \in C^{1+\alpha}(\Omega) \times\left(C^{2+\alpha}(\Omega)\right)^{3}
$$

satisfies the compatibility conditions, (1.10) and $v_{0}(x)>0, \theta_{0}(x)>0,0 \leq z_{0}(x) \leq 1$ for any $x \in[0,1]$. Then there exists a unique solution $(v, u, \theta, z)$ of the initial boundary value problem (1.1)-(1.4), (1.8)-(1.9) such that for any $T>0$,

$$
\left(v, v_{x}, v_{t}\right) \in\left(C_{x, t}^{\alpha, \alpha / 2}\left(Q_{T}\right)\right)^{3}, \quad(v, \theta, z) \in\left(C_{x, t}^{2+\alpha, 1+\alpha / 2}\left(Q_{T}\right)\right)^{3} .
$$


Moreover, for any $(x, t) \in \overline{Q_{T}}$,

$$
v(x, t)>0, \quad \theta(x, t)>0,0 \leq z(x, t) \leq 1 .
$$

In recent years, the heat-conducting radiative viscous gas has drawn the attention of mathematicians (see, e.g., [2]-[15, [21], 23], 24], 27, 28]). For the compressible viscous and heat-conducting model in one space dimension, the global existence and large-time behavior of smooth (strong, weak) solutions have been established by many authors. Among them, Antontsev, Kazhikhov and Monakhov [1, Chen [2, Kazhikhov and Shelukhin [15, Ducomet 4, Ducomet and Zlotnik [13-14] studied a one-dimensional gaseous model similar to ours, i.e., a radiative and reactive model with the free-boundary in the external force field. However, in a series of papers [4, 13], 14, they adopted as a self-gravitation, a special form independent-of-time variable explicitly in the Lagrangian mass coordinate system, not the exact form (see (1.8) in [28]), which is called the "pancakes models" relevant to some large-scale structure of the universe (see, e.g., 24]). Qin [20] established the global existence, exponential stability and the existence of global attractors for a $1 D$ viscous heat-conducting real gas. Moreover, we note the global existence of solutions to some initial boundary value problem (1.1)-(1.4), not the pure free-boundary case (1.8), but with partially Dirichlet boundary conditions. For $q \geq 4, \beta>0$, Ducomet [5] proved the global existence and exponential decay in $H^{1}$ of solutions to (1.1)-(1.4) with the boundary conditions

$$
\left.\left(u, \theta_{x}, z_{x}\right)\right|_{x=0,1}=0 .
$$

However, there exist some defects in the argumentation of the main results in 5. Recently, Qin et al. 22] corrected these defects in [5] and established the global existence and exponential stability of solutions in $H^{i}(i=1,2,4)$ to (1.1)-(1.4) with boundary condition (1.15), which has improved the range of $(q, \beta)$ in [5]. For our problem, Umehara and Tani [27] proved the global solvability of smooth solutions for $4 \leq q \leq 16$ and $0 \leq \beta \leq 13 / 2$. Later on, they further improved their results in $[28]$ with the larger range of $(q, \beta) \in E_{2}$ than that in [27]. Recently, Qin et al. 21] further improved the results in [27]-28] with a larger range for

$$
(q, \beta) \in\{(q, \beta): 9 / 4<q<3,0 \leq \beta<2 q+6\} \cup\{(q, \beta): 3 \leq q, 0 \leq \beta<q+9\}
$$

than those in [27]-28].

The aim of this paper is to further improve those results in [21, 27, 28, with the larger range of $(q, \beta) \in E=E_{1} \cup E_{2}$ than those in [21, 27]-28].

The main mathematical difficulty arises from the higher-order nonlinearities of the temperature $\theta$ in $p(v, \theta), e(v, \theta)$ and $\kappa(v, \theta)$ in (1.5)-(1.6). To overcome this difficulty, we shall first use the delicate interpolation techniques to reduce the order of $\theta$, and then bound the norms of $(v, u, \theta, z)$ and their derivatives in terms of expressions of the form

$$
A^{\Lambda} \equiv\left(\sup _{0 \leq s \leq t}\|\theta(s)\|_{L^{\infty}}\right)^{\Lambda}
$$

with $\Lambda$ being a positive constant depending only on $q$ and $\beta$. 
The local existence of solutions can be established by the standard method (see, e.g., [25]-[26]). Therefore to prove our results, it suffices to continue the local solutions by establishing the following a priori estimates.

ThEOREM 1.2. Let $T>0$ be an arbitrarily given constant. Under the assumptions of Theorem 1.1, we assume further that problem (1.1)-(1.4), (1.8)-(1.9) possesses a solution $(v, u, \theta, z)$ such that

$$
\left(v, v_{x}, v_{t}\right) \in\left(C_{x, t}^{\alpha, \alpha / 2}\left(Q_{T}\right)\right)^{3}, \quad(u, \theta, z) \in\left(C_{x, t}^{2+\alpha, 1+\alpha / 2}\left(Q_{T}\right)\right)^{3} .
$$

Then there exists a positive constant $C$ depending on the initial data and $T$ such that

$$
\left|\left(v, v_{x}, v_{t}\right)\right|_{\alpha, \alpha / 2}+|(u, \theta, z)|_{2+\alpha, 1+\alpha / 2} \leq C
$$

and for any $(x, t) \in \overline{Q_{T}}$,

$$
v(x, t), \theta(x, t) \geq C^{-1}>0,0 \leq z(x, t) \leq 1 .
$$

2. Proof of Theorem 1.1. To prove Theorem 1.1, we only need to prove Theorem 1.2. To this end, we shall establish several lemmas concerning the estimates of the solution and its derivatives. Our methods are mainly based on the techniques in Qin [20]-21]; that is, we estimate delicately the solution and its higher derivatives in terms of functions $A, X, Y$ and $Z$ (see their definitions below) and by means of the delicate interpolation techniques.

Now define the following functions:

$$
\begin{aligned}
& |u|^{(0)}:=\sup _{(x, t) \in Q_{T}}|u(x, t)|, \quad A=A(t):=\sup _{0 \leq s \leq t}\|\theta(s)\|_{L^{\infty}(\Omega)}, \\
& X=X(t):=\int_{0}^{t} \int_{0}^{1}\left(1+\theta^{q+3}\right) \theta_{t}^{2} d x d s, \quad Y=Y(T):=\max _{t \in[0, T]} \int_{0}^{1}\left(1+\theta^{2 q}\right) \theta_{x}^{2} d x, \\
& Z=Z(T):=\max _{t \in[0, T]}\left\|u_{x x}(t)\right\|^{2} .
\end{aligned}
$$

Lemma 2.1. For any $t \in[0, T]$, we have

$$
\begin{array}{r}
A \leq|\theta|^{(0)} \leq C+C Y^{\frac{1}{2 q+6}}, \\
\max _{t \in[0, T]}\left\|u_{x}\right\|^{2} \leq C+C Z^{\frac{1}{2}}, \\
\left|u_{x}\right|^{(0)} \leq C+C Z^{\frac{3}{8}}, \\
|u|^{(0)} \leq C+C Z^{\frac{1}{8}} .
\end{array}
$$

Proof. See, e.g., [27]-28].

Lemma 2.2. For any $t \in[0, T]$, we have

$$
\begin{gathered}
\int_{0}^{1}\left(\frac{1}{2} u^{2}+e+\lambda z+f(x) v\right) d x=E_{0} \\
U(t)+\int_{0}^{t} V(\tau) d \tau \leq C_{0}
\end{gathered}
$$




$$
\begin{gathered}
\int_{0}^{1} z d x+\int_{0}^{t} \int_{0}^{1} \phi z d x d \tau=\int_{0}^{1} z_{0} d x \\
\int_{0}^{1} \frac{1}{2} z^{2} d x+\int_{0}^{t} \int_{0}^{1}\left(\frac{d}{v^{2}} z_{x}^{2}+\phi z^{2}\right) d x d \tau=\int_{0}^{1} \frac{1}{2} z_{0}^{2} d x \\
\int_{0}^{t} \max _{x \in \bar{\Omega}} \theta^{r} d \tau \leq C, \quad 0 \leq r \leq q+4, q \geq 0 \\
\|u(t)\|^{2}+\int_{0}^{t}\left\|u_{x}(\tau)\right\|^{2} d \tau \leq C, \\
\left\|v_{x}(t)\right\|^{2}+\int_{0}^{t} \int_{0}^{1} \theta v_{x}^{2} d x d \tau \leq C, \quad q \geq 2, \\
\int_{0}^{t} \int_{0}^{1} u_{x}^{3} d x d \tau \leq C, \quad q \geq 4 \\
0 \leq z(x, t) \leq 1, \\
0<C^{-1} \leq v(x, t) \leq C
\end{gathered}
$$

where

$$
\begin{aligned}
E_{0} & :=\int_{0}^{1}\left(\frac{1}{2} u_{0}^{2}+e_{0}+\lambda z_{0}+f(x) v_{0}\right) d x \\
U(t) & :=\int_{0}^{1}\left[c_{V}(\theta-1-\log (\theta))+R(v-1-\log v)\right] d x \\
V(t) & :=\int_{0}^{1}\left(\frac{\mu u_{x}^{2}}{v \theta}+\frac{k \theta_{x}^{2}}{v \theta^{2}}+\frac{\lambda \phi}{\theta} z\right) d x \\
e_{0} & :=c_{V} \theta_{0}+a v_{0} \theta_{0}^{4}, f(x)=p_{e}+\frac{1}{2} G x(1-x) .
\end{aligned}
$$

Proof. For the proof of this lemma we mainly use the standard energy methods (see, e.g., [27]-28]).

From Lemma 2.2 we can easily obtain the following estimates (see, e.g., 21]).

Lemma 2.3. For any $t>0$, we have

$$
\begin{array}{r}
\int_{0}^{t}\|u\|_{L^{\infty}}^{2} d s \leq C, \\
\int_{0}^{1} \theta^{r} d x \leq C, \quad 0 \leq r \leq 4, \\
\int_{0}^{1} \frac{v \theta^{r}}{1+v \theta^{q}} d x \leq C, \quad 0 \leq r \leq q+4 .
\end{array}
$$

Proof. By the Gagliardo-Nirenberg interpolation inequality and (2.5), (2.10), we have

$$
\begin{aligned}
\int_{0}^{t}\|u(s)\|_{L^{\infty}(\Omega)}^{2} d s & \leq C \int_{0}^{t}\|u(s)\|\left\|u_{x}(s)\right\| d s \\
& \leq C \int_{0}^{t}\left\|u_{x}(s)\right\| d s \\
& \leq C .
\end{aligned}
$$


For $0 \leq r \leq 4$, by the Hölder inequality (2.5) and (2.13), we derive that

$$
\begin{aligned}
\int_{0}^{1} \theta^{r}(x, t) d x & \leq\left(\int_{0}^{1} \theta^{4}(x, t) d x\right)^{\frac{r}{4}}\left(\int_{0}^{1} 1 d x\right)^{1-\frac{r}{4}} \\
& \leq C\left(\int_{0}^{1} \theta^{4}(x, t) d x\right)^{\frac{r}{4}} \\
& \leq C .
\end{aligned}
$$

For $0 \leq r \leq q+4$, we infer from (2.13) and (2.15) that

$$
\begin{aligned}
\int_{0}^{1} \frac{v \theta^{r}}{1+v \theta^{q}} d x & \leq \int_{0}^{1}(1+\theta)^{r-q} d x \\
& \leq C
\end{aligned}
$$

The proof is now complete.

Lemma 2.4. If $q \geq 2,0 \leq m \leq \frac{q+4}{2}$, for $\forall t \in(0, T]$, the following inequalities hold:

$$
\begin{aligned}
& \int_{0}^{t} \int_{0}^{1}(1+\theta)^{2 m} v_{x}^{2} d x d s \leq C, \\
& \int_{0}^{t} \int_{0}^{1}(1+\theta)^{2 m} u^{2} d x d s \leq C .
\end{aligned}
$$

Proof. Since there exist constants $b(t) \in[0,1]$ and $r_{1}, r_{2}>0$, such that

$$
0<r_{1} \leq \int_{0}^{1} \theta(x, t) d x=\theta(b(t), t):=\theta_{1} \leq r_{2},
$$

by (2.6), (2.16) and the Hölder inequality, we deduce that for $0 \leq m \leq \frac{q+4}{2}$,

$$
\begin{aligned}
\left|\theta^{m}(x, t)-\theta_{1}^{m}\right| & \leq C \int_{0}^{1}\left|\theta^{m-1} \theta_{x}\right| d x \\
& \leq C(R(t))^{\frac{1}{2}}\left(\int_{0}^{1} \frac{v \theta^{2 m}}{k} d x\right)^{\frac{1}{2}} \\
& \leq C(R(t))^{\frac{1}{2}} .
\end{aligned}
$$

Here we denote $R(t):=\int_{0}^{1} \frac{k \theta_{x}^{2}}{v \theta^{2}} d x$, which implies

$$
C-C_{1} R(t) \leq \theta^{2 m}(x, t) \leq C+C_{1} R(t), \quad 0 \leq m \leq \frac{q+4}{2} .
$$

If $0 \leq m \leq \frac{q+4}{2}$, then by (2.6), (2.11) and (2.19), we have

$$
\begin{aligned}
\int_{0}^{t} \int_{0}^{1}(1+\theta)^{2 m} v_{x}^{2} d x d s & \leq C \int_{0}^{t} \int_{0}^{1} v_{x}^{2} d x d s+C \int_{0}^{t} R(s) \int_{0}^{1} v_{x}^{2} d x d d s \\
& \leq C+C \int_{0}^{t} R(s) d s \\
& \leq C, \\
\int_{0}^{t} \int_{0}^{1}(1+\theta)^{2 m} u^{2} d x d s & \leq C \int_{0}^{t} \int_{0}^{1} u^{2} d x d s+C \int_{0}^{t} \int_{0}^{1} u^{2} d x R(s) d s \\
& \leq C .
\end{aligned}
$$


Thus the proof is complete.

Lemma 2.5. If $q \geq 2$ and $0 \leq m \leq \frac{q+4}{2}$, for any $\varepsilon>0$ and any $t \in(0, T]$, the following estimates hold:

$$
\begin{gathered}
\left\|u_{x}(t)\right\|^{2}+\int_{0}^{t}\left\|u_{x x}(s)\right\|^{2} d s \leq C+\varepsilon X+C A^{q_{3}}+C Z^{\frac{2}{3}}, \\
\int_{0}^{t} \int_{0}^{1}(1+\theta)^{2 m} u_{x}^{2} d x d s \leq C+\varepsilon X+C A^{q_{3}}+C Z^{\frac{2}{3}},
\end{gathered}
$$

where $q_{1}=\max (8-q, 0), q_{2}=\max (3-q, 0), q_{3}=\max \left(q_{1}, 2 q_{2}, \frac{4}{3}\right)$.

Proof. Multiplying (1.2) by $u_{x x}$ and integrating it on $(0,1) \times(0, t), t \in(0, T]$, we arrive at

$$
\begin{aligned}
\int_{0}^{t} \int_{0}^{1} u_{t} u_{x x} d x d s & =\int_{0}^{t} \int_{0}^{1}\left(-p_{x} u_{x x}+\mu \frac{u_{x x}^{2}}{v}-\mu \frac{u_{x} v_{x} u_{x x}}{v^{2}}-G\left(x-\frac{1}{2}\right) u_{x x}\right) d x d s \\
& =\int_{0}^{t} \int_{0}^{1}\left(-p_{x} u_{x x}+\mu \frac{u_{x x}^{2}}{v}-\mu \frac{u_{x} v_{x} u_{x x}}{v^{2}}\right) d x d s-\int_{0}^{t} G\left(x-\frac{1}{2}\right) u_{x x} d s
\end{aligned}
$$

whence, by Lemmas 1.1-1.4,

$$
\begin{aligned}
\left\|u_{x}(t)\right\|^{2}+\int_{0}^{t}\left\|u_{x x}(s)\right\|^{2} d s \leq & C+C \int_{0}^{t}\left\|u_{t}\right\|_{L^{\infty}(\Omega)}\left\|u_{x}\right\|_{L^{\infty}(\Omega)} d s+C \int_{0}^{t}\left\|u_{x}\right\|_{L^{\infty}(\Omega)} d s \\
& +C \int_{0}^{t} \int_{0}^{1}\left[\left(1+\theta^{3}\right)\left|\theta_{x} u_{x x}\right|+\left|\theta v_{x} u_{x x}\right|+\left|u_{x x}\right|\right] d x d s .
\end{aligned}
$$

Using Lemmas 2.1-2.4, the Gagliardo-Nirenberg interpolation inequality, Young's inequality, and Hölder's inequality, we easily derive that for any $\varepsilon>0$,

$$
\begin{aligned}
& \int_{0}^{t} \int_{0}^{1}\left(1+\theta^{3}\right)\left|\theta_{x} u_{x x}\right| d x d s \leq \varepsilon \int_{0}^{t} \int_{0}^{1} u_{x x}^{2} d x d s+C \int_{0}^{t} \int_{0}^{1}\left(1+\theta^{3}\right)^{2} \theta_{x}^{2} d x d s \\
& \leq \varepsilon \int_{0}^{t} \int_{0}^{1} u_{x x}^{2} d x d s+C \sup _{0 \leq s \leq t}\left\|\frac{\left(1+\theta^{3}\right)^{2} \theta^{2}}{k}\right\|_{L^{\infty}(\Omega)} \int_{0}^{t} R(s) d s \\
& \leq \varepsilon \int_{0}^{t} \int_{0}^{1} u_{x x}^{2} d x d s+C+C A^{q_{1}}, \\
& \int_{0}^{t} \int_{0}^{1}\left|\theta v_{x} u_{x x}\right| d x d s \leq \varepsilon \int_{0}^{t} \int_{0}^{1} u_{x x}^{2} d x d s+C \int_{0}^{t} \int_{0}^{1} \theta^{2} v_{x}^{2} d x d s \\
& \leq \varepsilon \int_{0}^{t} \int_{0}^{1} u_{x x}^{2} d x d s+C, \\
& \int_{0}^{t} \int_{0}^{1}\left|u_{x} v_{x} u_{x x}\right| d x d s \leq \varepsilon \int_{0}^{t} \int_{0}^{1} u_{x x}^{2} d x d s+C \int_{0}^{t} \int_{0}^{1} u_{x}^{2} v_{x}^{2} d x d s \\
& \leq \varepsilon \int_{0}^{t} \int_{0}^{1} u_{x x}^{2} d x d s+C \int_{0}^{t}\left\|u_{x}\right\|_{L^{\infty}}^{2}\left\|v_{x}\right\|^{2} d s \\
& \leq \varepsilon \int_{0}^{t} \int_{0}^{1} u_{x x}^{2} d x d s+C \int_{0}^{t}\left\|u_{x}\right\|\left\|u_{x x}\right\| d s \\
& \leq 2 \varepsilon \int_{0}^{t} \int_{0}^{1} u_{x x}^{2} d x d s+C,
\end{aligned}
$$




$$
\begin{aligned}
& \int_{0}^{t} \int_{0}^{1}\left|u_{x x}\right| d x d s \leq \varepsilon \int_{0}^{t}\left\|u_{x x}\right\|^{2} d s+C \\
& \int_{0}^{t}\left\|u_{t}\right\|_{L^{\infty}}\left\|u_{x}\right\|_{L^{\infty}} d s \leq C \int_{0}^{t}\left(\left\|u_{t}\right\|^{\frac{1}{2}}\left\|u_{x t}\right\|^{\frac{1}{2}}+\left\|u_{t}\right\|\right)\left(\left\|u_{x}\right\|^{\frac{1}{2}}\left\|u_{x x}\right\|^{\frac{1}{2}}+\left\|u_{x}\right\|\right) d s \\
& \leq C\left(\int_{0}^{t}\left(\left\|u_{x t}\right\|\left\|u_{t}\right\|+\left\|u_{t}\right\|^{2}\right) d s\right)^{\frac{1}{2}}\left(\int_{0}^{t}\left(\left\|u_{x}\right\|\left\|u_{x x}\right\|+\left\|u_{x}\right\|^{2}\right) d s\right)^{\frac{1}{2}} \\
& \leq C\left(\left(\int_{0}^{t}\left\|u_{x}\right\|^{2} d s\right)^{\frac{1}{4}}\left(\int_{0}^{t}\left\|u_{x x}\right\|^{2} d s\right)^{\frac{1}{4}}+\left(\int_{0}^{t}\left\|u_{x}\right\|^{2} d s\right)^{\frac{1}{2}}\right) \\
& \times\left(\left(\int_{0}^{t}\left\|u_{x t}\right\|^{2} d s\right)^{\frac{1}{4}}\left(\int_{0}^{t}\left\|u_{t}\right\|^{2} d s\right)^{\frac{1}{4}}+\left(\int_{0}^{t}\left\|u_{t}\right\|^{2} d s\right)^{\frac{1}{2}}\right) \\
& \leq \varepsilon \int_{0}^{t}\left\|u_{x x}\right\|^{2} d s+C\left(\left(\int_{0}^{t}\left\|u_{x t}\right\|^{2} d s\right)^{\frac{1}{4}}\left(\int_{0}^{t}\left\|u_{t}\right\|^{2} d s\right)^{\frac{1}{4}}+\left(\int_{0}^{t}\left\|u_{t}\right\|^{2} d s\right)^{\frac{1}{2}}\right)^{\frac{4}{3}} \\
& +C\left(\left(\int_{0}^{t}\left\|u_{x t}\right\|^{2} d s\right)^{\frac{1}{4}}\left(\int_{0}^{t}\left\|u_{t}\right\|^{2} d s\right)^{\frac{1}{4}}+\left(\int_{0}^{t}\left\|u_{t}\right\|^{2} d s\right)^{\frac{1}{2}}\right) \\
& \leq \varepsilon \int_{0}^{t}\left\|u_{x x}\right\|^{2} d s+C+C\left(\int_{0}^{t}\left\|u_{x t}\right\|^{2} d s\right)^{\frac{1}{3}}\left(\int_{0}^{t}\left\|u_{t}\right\|^{2} d s\right)^{\frac{1}{3}} \\
& +C\left(\int_{0}^{t}\left\|u_{t}\right\|^{2} d s\right)^{\frac{2}{3}} \\
& \leq \varepsilon \int_{0}^{t}\left\|u_{x x}\right\|^{2} d s+C+C\left(\int_{0}^{t}\left\|u_{x t}\right\|^{2} d s\right)^{\frac{1}{3}} \sup _{0 \leq s \leq t}\left\|u_{t}(s)\right\|^{2 / 3} \\
& +C \sup _{0 \leq s \leq t}\left\|u_{t}(s)\right\|^{4 / 3} .
\end{aligned}
$$

In order to estimate the inequality (2.27), we differentiate (1.2) with respect to $t$, multiply it by $u_{t}$ and integrate it with respect to $x$. Then we have

$$
\frac{d}{d t} \int_{0}^{1} \frac{1}{2} u_{t}^{2} d x+\int_{0}^{1} \frac{\mu}{v} u_{x t}^{2} d x=\int_{0}^{1}\left(p_{t} u_{x t}+\frac{\mu}{v} u_{x}^{2} u_{x t}\right) d x
$$

which gives

$$
\begin{aligned}
\left\|u_{t}(t)\right\|^{2}+\int_{0}^{t}\left\|u_{x t}\right\|^{2} d s & \leq C+C \int_{0}^{t} \int_{0}^{1}\left(\left|p_{t} u_{x t}\right|+\left|u_{x}^{2} u_{x t}\right|\right) d x d s \\
& \leq \varepsilon \int_{0}^{t}\left\|u_{x t}(s)\right\|^{2} d s+C \int_{0}^{t} \int_{0}^{1}\left(p_{t}^{2}+u_{x}^{4}\right) d x d s+C .
\end{aligned}
$$

Here $\varepsilon>0$ is small enough.

Note the following facts:

$$
\begin{aligned}
\int_{0}^{t} \int_{0}^{1} p_{t}^{2} d x d s & \leq C \int_{0}^{t} \int_{0}^{1}\left[\left(1+\theta^{3}\right)^{2} \theta_{t}^{2}+\left|\theta u_{x}\right|^{2}\right] d x d s \\
& \leq C(1+A)^{q_{2}} X+C+C A^{2} \int_{0}^{t} \int_{0}^{1} u_{x}^{2} d x d s \\
& \leq C(1+A)^{q_{2}} X+C A^{2}+C,
\end{aligned}
$$




$$
\begin{aligned}
\int_{0}^{t} \int_{0}^{1} u_{x}^{4} d x d s & \leq C \int_{0}^{t}\left(\left\|u_{x}\right\|^{3}\left\|u_{x x}\right\|+\left\|u_{x}\right\|^{4}\right) d x d s \\
& \leq \varepsilon \int_{0}^{t}\left\|u_{x x}\right\|^{2} d s+C \int_{0}^{t}\left\|u_{x}\right\|^{6} d s \\
& \leq \varepsilon \int_{0}^{t}\left\|u_{x x}\right\|^{2} d s+C \max _{t \in[0, T]}\left\|u_{x}\right\|^{4} \int_{0}^{t}\left\|u_{x}\right\|^{2} d s \\
& \leq \varepsilon \int_{0}^{t}\left\|u_{x x}\right\|^{2} d s+C+C Z .
\end{aligned}
$$

We insert (2.29)-(2.30) into (2.28) to derive

$$
\sup _{0 \leq s \leq t}\left\|u_{t}(s)\right\|^{2}+\int_{0}^{t}\left\|u_{x t}\right\|^{2} d s \leq \varepsilon \int_{0}^{t}\left\|u_{x x}\right\|^{2} d s+C+C Z+C A^{2}+C(1+A)^{q_{2}} X,
$$

which, inserted into (2.27), implies that

$$
\int_{0}^{t}\left\|u_{t}\right\|_{L^{\infty}}\left\|u_{x}\right\|_{L^{\infty}} d s \leq 2 \varepsilon \int_{0}^{t}\left\|u_{x x}\right\|^{2} d s+C+C A^{\frac{4}{3}}+C Z^{\frac{2}{3}}+\varepsilon X+C A^{2 q_{2}} .
$$

From (2.23)-(2.26) and (2.32), we can easily get the inequality (2.20). By (2.20) we can easily prove the inequality $(2.21)$.

LEmma 2.6. If $q>2$, then the following inequality holds:

$$
\left\|\theta+\theta^{4}\right\|^{2}+\int_{0}^{t} \int_{0}^{1}(1+\theta)^{q+3} \theta_{x}^{2} d x d s \leq C+C A^{q_{6}}+\varepsilon X^{\frac{1}{2}}+C Z^{\frac{1}{3}}
$$

where $q_{0}=\max (4-q, 0), q_{4}=\max \left(7-2 q, q_{0}\right), q_{*}=\max ((12-q) / 2,0)$, and $q_{6}=$ $\max \left(q_{4}, q_{3} / 2,4, q_{*}\right)$.

Proof. Multiplying (1.3) by $e$ and integrating the resulting equation over $Q_{t} \equiv(0,1) \times$ $(0, t)$, we arrive at

$$
\begin{aligned}
\frac{1}{2}\|e\|^{2}= & \frac{1}{2}\left\|e_{0}\right\|^{2}+\left.\int_{0}^{t}(-p e) u\right|_{0} ^{1} d s-\int_{0}^{t} \int_{0}^{1}(-p e)_{x} u d x d s+\int_{0}^{t} \int_{0}^{1} \mu \frac{u_{x}^{2} e}{v} d x d s \\
& -\int_{0}^{t} \int_{0}^{1} \frac{k \theta_{x}}{v} e_{x} d x d s+\int_{0}^{t} \int_{0}^{1} \lambda \phi z e d x d s,
\end{aligned}
$$

whence

$$
\begin{aligned}
\|e\|^{2}+\int_{0}^{t} \int_{0}^{1}(1+\theta)^{q+3} \theta_{x}^{2} d x d s \leq & C+C \int_{0}^{t} \int_{0}^{1}\left[\left|(p e)_{x} u\right|+\left|e u_{x}^{2}\right|+\left|k \theta_{x} \theta^{4} v_{x}\right|\right. \\
& +|\lambda \phi z e|] d x d s+\int_{0}^{t}\|p e u\|_{L^{\infty}} d s .
\end{aligned}
$$

By Lemmas 2.1-2.5, Young's inequality and the Gagliardo-Nirenberg interpolation inequality, we infer that for any $\varepsilon>0$,

$$
\begin{aligned}
\int_{0}^{t} \int_{0}^{1}\left|(p e)_{x} u\right| d x d s & \leq C \int_{0}^{t} \int_{0}^{1}\left[\left(1+\theta^{7}\right)\left|\theta_{x} u\right|+\left(1+\theta^{5}\right)\left|v_{x} u\right|+\left(1+\theta^{8}\right)\left|v_{x} u\right|\right] d x d s \\
& \leq \varepsilon \int_{0}^{t} \int_{0}^{1}(1+\theta)^{q+3} \theta_{x}^{2} d x d s+C \int_{0}^{t} \int_{0}^{1}\left[(1+\theta)^{11-q} u^{2}\right.
\end{aligned}
$$




$$
\begin{aligned}
& \text { GLOBAL SMOOTH SOLUTIONS } \\
& \left.+\left(1+\theta^{5}\right)\left|v_{x} u\right|+\left(1+\theta^{8}\right)\left|v_{x} u\right|\right] d x d s \\
& \leq \varepsilon \int_{0}^{t} \int_{0}^{1}(1+\theta)^{q+3} \theta_{x}^{2} d x d s+C+C A^{\max (7-2 q, 0)} \\
& +C \int_{0}^{t} \int_{0}^{1}(1+\theta)^{8} v_{x}^{2} d x d s+C \int_{0}^{t} \int_{0}^{1}(1+\theta)^{8} u^{2} d x d s \\
& \leq \varepsilon \int_{0}^{t} \int_{0}^{1}(1+\theta)^{q+3} \theta_{x}^{2} d x d s+C+C A^{\max (7-2 q, 0)}+C A^{q_{0}} \\
& \leq \varepsilon \int_{0}^{t} \int_{0}^{1}(1+\theta)^{q+3} \theta_{x}^{2} d x d s+C A^{q_{4}}+C, \\
& \int_{0}^{t} \int_{0}^{1}\left|e u_{x}^{2}\right| d x d s \leq C \int_{0}^{t}\left\|u_{x}\right\|_{L^{\infty}}^{2} \int_{0}^{1} e d x d s \\
& \leq C \int_{0}^{t}\left\|u_{x}\right\|_{L^{\infty}}^{2} d s \\
& \leq C \int_{0}^{t}\left(\left\|u_{x}\right\|\left\|u_{x x}\right\|+\left\|u_{x}\right\|^{2}\right) d s \\
& \leq C\left(\int_{0}^{t}\left\|u_{x x}\right\|^{2} d s\right)^{\frac{1}{2}}+C \\
& \leq C+\varepsilon X^{\frac{1}{2}}+C A^{\frac{q_{3}}{2}}+C Z^{\frac{1}{3}}, \\
& \int_{0}^{t} \int_{0}^{1}\left|k \theta_{x} \theta^{4} v_{x}\right| d x d s \leq C \int_{0}^{t} \int_{0}^{1}(1+\theta)^{q+4}\left|\theta_{x} v_{x}\right| d x d s \\
& \leq \varepsilon \int_{0}^{t} \int_{0}^{1}(1+\theta)^{q+3} \theta_{x}^{2} d x d s+C \int_{0}^{t} \int_{0}^{1}(1+\theta)^{q+5} v_{x}^{2} d x d s \\
& \leq \varepsilon \int_{0}^{t} \int_{0}^{1}(1+\theta)^{q+3} \theta_{x}^{2} d x d s+C+C A \\
& \int_{0}^{t} \int_{0}^{1}|\lambda \phi z e| d x d s \leq C \int_{0}^{t} \int_{0}^{1} \phi e d x d s \\
& \leq C \sup _{0 \leq s \leq t}\|e\|_{L^{\infty}} \int_{0}^{t} \int_{0}^{1} \phi d x d s \\
& \leq C+C A^{4} \\
& \int_{0}^{t} \int_{0}^{1}\|p e u\|_{L^{\infty}} d x d s \leq\left(\int_{0}^{t}\|u\|_{L^{\infty}}^{2} d s\right)^{\frac{1}{2}}\left(\int_{0}^{t}\|p e\|_{L^{\infty}}^{2} d s\right)^{\frac{1}{2}} \\
& \leq C\left(\int_{0}^{t}\|p e\|_{L^{\infty}}^{2} d s\right)^{\frac{1}{2}} \\
& \leq C\left(\int_{0}^{t}\left\|\left(1+\theta^{4}\right)^{2}\right\|_{L^{\infty}}^{2} d s\right)^{\frac{1}{2}} \\
& \leq C+C A^{q_{*}} .
\end{aligned}
$$

Inserting (2.35)-(2.39) into (2.34), we can readily get the inequality (2.33). 
Next we denote the function $H$ by the following:

$$
H=H(v, \theta):=\int_{0}^{\theta} \frac{k(v, \xi)}{v} d \xi .
$$

Since (1.3) is rewritten as

$$
e_{\theta} \theta_{t}+\theta p_{\theta} u_{x}=\frac{\mu}{v} u_{x}^{2}+\left(\frac{k}{v} \theta_{x}\right)_{x}+\lambda \phi z,
$$

multiplying (2.39) by $H_{t}$ and integrating it over $\bar{\Omega} \times[0, t]$ yields

$$
\int_{0}^{t} \int_{0}^{1} e_{\theta} \theta_{t} H_{t} d x d s+\int_{0}^{t} \int_{0}^{1} \frac{k}{v} \theta_{x} H_{x t} d x d s=\int_{0}^{t} \int_{0}^{1}\left(-\theta p_{\theta} u_{x}+\frac{\mu}{v} u_{x}^{2}+\lambda \phi z\right) H_{t} d x d s .
$$

Here

$$
\begin{aligned}
& H_{t}=H_{v} u_{x}+\frac{k}{v} \theta_{t}, \\
& H_{x t}=\left(\frac{k \theta_{x}}{v}\right)_{t}+H_{v} u_{x x}+H_{v v} v_{x} u_{x}+\left(\frac{k}{v}\right)_{v} v_{x} \theta_{t}, \\
& \left|H_{v}\right|,\left|H_{v v}\right| \leq C \theta .
\end{aligned}
$$

Lemma 2.7. If $2 \leq q<3$ and $0 \leq \beta<2 q+6$ or $q \geq 3$ and $0 \leq \beta<q+9$, then there exists a number $\delta, 0<\delta<1$ such that

$$
X+Y \leq C\left(1+Z^{\delta}\right) .
$$

Proof. From (2.13) and the definitions of $X$ and $Y$, we can immediately derive the inequalities

$$
\begin{aligned}
\int_{0}^{t} \int_{0}^{1} e_{\theta} \theta_{t} \frac{k}{v} \theta_{t} d x d s & =\int_{0}^{t} \int_{0}^{1}\left(c_{V}+4 a v \theta^{3}\right) \frac{k_{1}+k_{2} v \theta^{q}}{v} \theta_{t}^{2} d x d s \\
& \geq C X, \\
\int_{0}^{t} \int_{0}^{1} \frac{k \theta_{x}}{v}\left(\frac{k \theta_{x}}{v}\right)_{t} d x d s & =\left.\frac{1}{2} \int_{0}^{1}\left(\frac{k \theta_{x}}{v}\right)^{2}\right|_{0} ^{t} d x \\
& \geq C Y-C .
\end{aligned}
$$

In the same way as that in 22, by Young's inequality and Hölder's inequality, for any $\varepsilon>0$, it is not difficult to get the following estimates by using Lemmas 1.1-1.6:

$$
\begin{aligned}
\int_{0}^{t} \int_{0}^{1} e_{\theta} \theta_{t} H_{v} u_{x} d x d s & \leq C \int_{0}^{t} \int_{0}^{1}(1+\theta)^{4} \theta_{t} u_{x} d x d s \\
& \leq \varepsilon X+C \int_{0}^{t} \int_{0}^{1}(1+\theta)^{5-q} u_{x}^{2} d x d s \\
& \leq \varepsilon X+C+C A^{q_{3}}+C Z^{\frac{2}{3}} \\
& \leq \varepsilon(X+Y)+C+C Z^{\frac{2}{3}} \\
\int_{0}^{t} \int_{0}^{1}\left(\theta p_{\theta} u_{x}-\frac{\mu}{v} u_{x}^{2}\right) H_{v} u_{x} d x d s & \leq C \int_{0}^{t} \int_{0}^{1}\left[\left(1+\theta^{5}\right) u_{x}^{2}+\theta u_{x}^{3}\right] d x d s
\end{aligned}
$$




$$
\begin{aligned}
\leq & C+\varepsilon X+C A^{q_{3}}+C Z^{\frac{2}{3}}+C \int_{0}^{t} \int_{0}^{1} \theta u_{x}^{3} d x d s \\
\leq & C+C A^{q_{3}}+C Z^{\frac{2}{3}}+\varepsilon X+C A\left|u_{x}\right|^{(0)} \\
\leq & C+C A^{q 3}+C Z^{\frac{2}{3}}+\varepsilon X+C A+C Z^{\frac{3}{8}} A \\
\leq & \varepsilon(X+Y)+C+C Z^{\frac{2}{3}} \\
\int_{0}^{t} \int_{0}^{1}\left(\theta p_{\theta} u_{x}-\frac{\mu}{v} u_{x}^{2}\right) \frac{k}{v} \theta_{t} d x d s \leq & C \int_{0}^{t} \int_{0}^{1}(1+\theta)^{q+4}\left|\theta_{t} u_{x}\right| d x d s \\
& +C \int_{0}^{t} \int_{0}^{1}(1+\theta)^{q}\left|\theta_{t} u_{x}^{2}\right| d x d s \\
\leq & \varepsilon X+C \int_{0}^{t} \int_{0}^{1}\left[(1+\theta)^{q+5} u_{x}^{2}+(1+\theta)^{q-3} u_{x}^{4}\right] d x d s \\
\leq & \varepsilon X+C(1+A)^{q+5}+C \int_{0}^{t} \int_{0}^{1}(1+\theta)^{q-3} u_{x}^{4} d x d s \\
\leq & \varepsilon(X+Y)+C+C \int_{0}^{t} \int_{0}^{1}(1+\theta)^{q-3} u_{x}^{4} d x d s
\end{aligned}
$$

which gives, for $2 \leq q<3$,

$$
\begin{aligned}
\int_{0}^{t} \int_{0}^{1}\left(\theta p_{\theta} u_{x}-\frac{\mu}{v} u_{x}^{2}\right) \frac{k}{v} \theta_{t} d x d s & \leq C+\varepsilon(X+Y)+C \int_{0}^{t} \int_{0}^{1}(1+\theta)^{q-3} u_{x}^{4} d x d s \\
& \leq \varepsilon(X+Y)+C+C \int_{0}^{t} \int_{0}^{1} u_{x}^{4} d x d s \\
& \leq \varepsilon(X+Y)+C+C\left|u_{x}^{2}\right|^{(0)} \int_{0}^{t} \int_{0}^{1} u_{x}^{2} d x d s \\
& \leq \varepsilon(X+Y)+C+C Z^{\frac{3}{4}}
\end{aligned}
$$

or for $3 \leq q<4$,

$$
\begin{aligned}
\int_{0}^{t} \int_{0}^{1}\left(\theta p_{\theta} u_{x}-\frac{\mu}{v} u_{x}^{2}\right) \frac{k}{v} \theta_{t} d x d s & \leq C+\varepsilon(X+Y)+C \int_{0}^{t} \int_{0}^{1}(1+\theta)^{q-3} u_{x}^{4} d x d s \\
& \leq \varepsilon(X+Y)+C+C \int_{0}^{t} \int_{0}^{1}(1+\theta) u_{x}^{4} d x d s \\
& \leq \varepsilon(X+Y)+C+C(1+A)\left|u_{x}^{2}\right|^{(0)} \int_{0}^{t} \int_{0}^{1} u_{x}^{2} d x d s \\
& \leq \varepsilon(X+Y)+C+C Z^{\frac{3}{4}}+C A+C A Z^{\frac{3}{4}} \\
& \leq \varepsilon(X+Y)+C+C Z^{\frac{3(q+3)}{2(2 q+5)}}
\end{aligned}
$$

or for $q \geq 4$, 


$$
\begin{aligned}
\int_{0}^{t} \int_{0}^{1}\left(\theta p_{\theta} u_{x}-\frac{\mu}{v} u_{x}^{2}\right) \frac{k}{v} \theta_{t} d x d s \leq & \varepsilon(X+Y)+C+C \int_{0}^{t} \int_{0}^{1}(1+\theta)^{q-3} u_{x}^{4} d x d s \\
\leq & \varepsilon(X+Y)+C+C(1+A)^{q-3}\left|u_{x}\right|^{(0)} \int_{0}^{t} \int_{0}^{1} u_{x}^{3} d x d s \\
\leq & \varepsilon(X+Y)+C+C(1+A)^{q-3} Z^{\frac{3}{8}}+C Z^{\frac{3}{8}} \\
\leq & \varepsilon(X+Y)+C+C Z^{\frac{3(q+3)}{4(q+9)}} \\
\int_{0}^{t} \int_{0}^{1} \frac{k \theta_{x}}{v} H_{v} u_{x x} d x d s \leq & C \int_{0}^{t} \int_{0}^{1}(1+\theta)^{q+1}\left|\theta_{x} u_{x x}\right| d x d s \\
\leq & C\left(\int_{0}^{t} \int_{0}^{1}(1+\theta)^{q+3} \theta_{x}^{2} d x d s\right)^{\frac{1}{2}} \\
& \times\left(\int_{0}^{t} \int_{0}^{1}(1+\theta)^{q-1} u_{x x}^{2} d x d s\right)^{\frac{1}{2}} \\
\leq & C\left(\int_{0}^{t} \int_{0}^{1}(1+\theta)^{q+3} \theta_{x}^{2} d x d s\right)^{\frac{1}{2}} \\
& \times\left(\max _{t \in[0, T]}\left\|u_{x x}\right\|^{2} \int_{0}^{t} \max _{x \in \bar{\Omega}}(1+\theta)^{q-1} d s\right)^{\frac{1}{2}} \\
\leq & C\left(1+A^{q 6}+Z^{\frac{1}{3}}+\varepsilon X^{\frac{1}{2}}\right)^{\frac{1}{2}} Z^{\frac{1}{2}} \\
\leq & C Z^{\frac{1}{2}}+C A^{\frac{q_{6}}{2}} Z^{\frac{1}{2}}+\varepsilon X^{\frac{1}{4}} Z^{\frac{1}{2}}+C Z^{\frac{2}{3}} \\
\leq & \varepsilon(X+Y)+C+C Z^{\delta^{\prime}} .
\end{aligned}
$$

Here we require

$$
q_{6}<2 q+6
$$

in order to make $A^{\frac{q_{6}}{2}}<\varepsilon Y+C$, and $0<\delta^{\prime}=\max \left(\frac{2}{3}, \frac{q+3}{2 q+6-q_{6} / 2}\right)<1$ hold.

Similarly,

$$
\begin{aligned}
\int_{0}^{t} \int_{0}^{1} \frac{k \theta_{x}}{v} H_{v v} v_{x} u_{x} d x d s \leq & C \int_{0}^{t} \int_{0}^{1}(1+\theta)^{q+1}\left|\theta_{x} v_{x} u_{x}\right| d x d s \\
\leq & C\left(\int_{0}^{t} \int_{0}^{1}(1+\theta)^{q+3} \theta_{x}^{2} d x d s\right)^{\frac{1}{2}} \\
& \times\left(\int_{0}^{t} \int_{0}^{1}(1+\theta)^{q-1} v_{x}^{2} u_{x}^{2} d x d s\right)^{\frac{1}{2}} \\
\leq & C\left(\int_{0}^{t} \int_{0}^{1}(1+\theta)^{q+3} \theta_{x}^{2} d x d s\right)^{\frac{1}{2}} \\
& \times\left(\left|u_{x}^{2}\right|^{(0)} \int_{0}^{t} \int_{0}^{1}(1+\theta)^{q-1} v_{x}^{2} d x d s\right)^{\frac{1}{2}} \\
\leq & C\left(1+A^{q_{6}}+Z^{\frac{1}{3}}+\varepsilon X^{\frac{1}{2}}\right)^{\frac{1}{2}}\left(1+Z^{\frac{3}{8}}\right)
\end{aligned}
$$




$$
\begin{aligned}
\leq & C+C A^{\frac{q_{6}}{2}}+C Z^{\frac{1}{6}}+\varepsilon X^{\frac{1}{4}}+C Z^{\frac{3}{8}}+C Z^{\frac{3}{8}} A^{\frac{q_{6}}{2}} \\
& +C Z^{\frac{13}{24}}+\varepsilon X^{\frac{1}{4}} Z^{\frac{3}{8}} \\
\leq & \varepsilon(X+Y)+C+C Z^{\delta_{1}},
\end{aligned}
$$

where $\delta_{1}=\max \left(\frac{13}{24}, \frac{3(q+3)}{4\left(2 q+6-q_{6} / 2\right)}\right)$. By $(2.51)$, we know that $q_{6} / 2<2 q+6$ and $A^{q_{6} / 2}<$ $\varepsilon Y+C, 0<\delta_{1}<1$.

By (2.39), we have

$$
\left(\frac{k \theta_{x}}{v}\right)_{x}=e_{\theta} \theta_{t}+\theta p_{\theta} u_{x}-\mu \frac{u_{x}^{2}}{v}-\lambda \phi z,
$$

which gives

$$
\begin{aligned}
\int_{0}^{t}\left\|\left(\frac{k \theta_{x}}{v}\right)_{x}\right\|^{2} d s & \leq C \int_{0}^{t}\left[\left\|e_{\theta} \theta_{t}\right\|^{2}+\left\|\theta p_{\theta} u_{x}\right\|^{2}+\left\|u_{x}^{2}\right\|^{2}+\|\phi\|^{2}\right] d s \\
& \leq C \int_{0}^{t} \int_{0}^{1}\left[(1+\theta)^{6} \theta_{t}^{2}+(1+\theta)^{8} u_{x}^{2}+u_{x}^{4}+\phi^{2}\right] d x d s \\
& \leq C(1+A)^{q_{2}} X+C+C A^{8}+C A^{\beta}+C\left|u_{x}^{2}\right|^{(0)} \int_{0}^{t} \int_{0}^{1} u_{x}^{2} d x d s \\
& \leq C+C(1+A)^{q_{2}} X+C A^{8}+C A^{\beta}+C Z^{\frac{3}{4}} .
\end{aligned}
$$

Here we have used the fact that

$$
\begin{aligned}
\int_{0}^{t} \int_{0}^{1} \phi^{2} d x d s & \leq C A^{\beta} \int_{0}^{t} \int_{0}^{1} \phi d x d s \\
& \leq C+C A^{\beta} .
\end{aligned}
$$

Then we infer from (2.52),

$$
\begin{aligned}
\int_{0}^{t} \int_{0}^{1} \frac{k \theta_{x}}{v}\left(\frac{k}{v}\right)_{v} v_{x} \theta_{t} d x d s \leq & C \int_{0}^{t} \int_{0}^{1}(1+\theta)^{q}\left|\theta_{x} v_{x} \theta_{t}\right| d x d s \\
\leq & \varepsilon X+C \int_{0}^{t}\left\|\frac{k \theta_{x}}{v}\right\|_{L^{\infty}}^{2}\left(\int_{0}^{1} \frac{(1+\theta)^{q-3}}{k^{2}} v_{x}^{2} d x\right) d s \\
\leq & \varepsilon X+C \int_{0}^{t}\left\|\frac{k \theta_{x}}{v}\right\|\left\|\left(\frac{k \theta_{x}}{v}\right)_{x}\right\| d s \\
\leq & \varepsilon X+C(1+A)^{q_{7} / 2}\left(\int_{0}^{t} \int_{0}^{1}(1+\theta)^{q+3} \theta_{x}^{2} d x d s\right)^{\frac{1}{2}} \\
& \times\left(\int_{0}^{t}\left\|\left(\frac{k \theta_{x}}{v}\right)_{x}\right\|^{2} d s\right)^{\frac{1}{2}} \\
\leq & \varepsilon X+C(1+A)^{\frac{q_{7}}{2}}\left(C+C A^{q_{6}}+\varepsilon X^{\frac{1}{2}}+C Z^{\frac{1}{3}}\right)^{\frac{1}{2}} \\
& \times\left(C+C(1+A)^{q_{2}} X+C A^{8}+C A^{\beta}+C Z^{\frac{3}{4}}\right)^{\frac{1}{2}} \\
\leq & \varepsilon X+\left(C+C A^{\frac{q_{7}}{2}}\right)\left(C+C A^{\frac{q_{6}}{2}}+\varepsilon X^{\frac{1}{4}}+C Z^{\frac{1}{6}}\right) \\
& \times\left(C+C(1+A)^{\frac{q_{2}}{2}} X^{\frac{1}{2}}+C A^{4}+C A^{\frac{\beta}{2}}+C Z^{\frac{3}{8}}\right)
\end{aligned}
$$




$$
\begin{aligned}
\leq & \varepsilon X+\left(C+\varepsilon X^{\frac{1}{4}} A^{\frac{q_{7}}{2}}+C A^{\frac{q_{6}+q_{7}}{2}}+C A^{\frac{q_{7}}{2}} Z^{\frac{1}{6}}\right) \\
& \times\left(C+C(1+A)^{\frac{q_{2}}{2}} X^{\frac{1}{2}}+C A^{4}+C A^{\frac{\beta}{2}}+C Z^{\frac{3}{8}}\right) \\
\leq & 2 \varepsilon X+\left(A^{2\left(q_{2}+q_{7}\right)}+A^{\frac{4}{3}\left(4+\frac{q_{7}}{2}\right)}+A^{\frac{4}{3}\left(\frac{\beta+q_{7}}{2}\right)}+A^{q_{2}+q_{6}+q_{7}}\right. \\
& \left.+A^{4+\frac{q_{6}+q_{7}}{2}}+A^{\frac{\beta+q_{6}+q_{7}}{2}}\right)+\left(Z^{\frac{13}{24}}+A^{\frac{2 q_{7}}{3}} Z^{\frac{1}{2}}+A^{q_{2}+q_{7}} Z^{\frac{1}{3}}\right. \\
& \left.+A^{4+\frac{q_{7}}{2}} Z^{\frac{1}{6}}+A^{\frac{q_{7}+\beta}{2}} Z^{\frac{1}{6}}+A^{\frac{q_{7}}{2}} Z^{\frac{13}{24}}\right) \\
\leq & 2 \varepsilon(X+Y)+C+C Z^{\delta_{2}},
\end{aligned}
$$

where $q_{7}=\max (q-3,0)$, and

$$
\begin{aligned}
\delta_{2}=\max \left(\frac{13}{24}, \frac{q+3}{2 q+6-\frac{2 q_{7}}{3}},\right. & \frac{2 q+6}{3\left(2 q+6-q_{2}-q_{7}\right)}, \frac{q+3}{3\left(2 q+2-\frac{q_{7}}{2}\right)}, \\
& \left.\frac{q+3}{3\left(2 q+6-\frac{\beta+q_{7}}{2}\right)}, \frac{13(q+3)}{12\left(2 q+6-\frac{q_{7}}{2}\right)}\right) .
\end{aligned}
$$

Here in order to make (2.54) hold and $0<\delta_{2}<1$, we must demand

$$
\left\{\begin{array}{l}
0 \leq q_{2}+q_{6}+q_{7}<2 q+6, \\
0 \leq 4+\frac{q_{6}+q_{7}}{2}<2 q+6, \\
0 \leq \frac{\beta+q_{6}+q_{7}}{2}<2 q+6 \\
0 \leq 2\left(q_{2}+q_{7}\right)<2 q+6 \\
0 \leq \frac{4}{3}\left(4+\frac{q_{7}}{2}\right)<2 q+6, \\
0 \leq \frac{4}{3} \frac{\beta+q_{7}}{2}<2 q+6
\end{array}\right.
$$

that is,

$$
\left\{\begin{array}{l}
0 \leq q_{2}+q_{6}+q_{7}<2 q+6 \\
0 \leq q_{6}+q_{7}<4 q+4 \\
0 \leq q_{6}+q_{7}+\beta<4 q+12 \\
0 \leq q_{2}+q_{7}<q+3 \\
0 \leq q_{7}<3 q+1 \\
0 \leq q_{7}+\beta<3 q+9
\end{array}\right.
$$

or

$$
\left\{\begin{array}{l}
0 \leq q_{7}<\frac{3}{2}(q+3) \\
0 \leq \beta+q_{7}<\frac{10}{3}(q+3) \\
0 \leq q_{2}+q_{7}<\frac{4}{3}(q+3)
\end{array}\right.
$$

and

$$
\left\{\begin{array}{l}
0 \leq \frac{q+3}{2 q+6-\frac{2}{3} q_{7}}<1, \\
0 \leq \frac{2 q+6}{3\left(2 q+6-q_{2}-q_{7}\right)}<1, \\
0 \leq \frac{q+3}{3\left(2 q+2-\frac{q_{7}}{2}\right)}<1, \\
0 \leq \frac{q+3}{3\left(2 q+6-\frac{q_{7}+\beta}{2}\right)}<1, \\
0 \leq \frac{13(q+3)}{12\left(2 q+6-\frac{q_{7}}{2}\right)}<1 .
\end{array}\right.
$$


Similarly,

$$
\begin{aligned}
\int_{0}^{t} \int_{0}^{1} \lambda \phi z H_{t} d x d s \leq & C \int_{0}^{t} \int_{0}^{1} \phi\left|H_{v} u_{x}+\frac{k}{v} \theta_{t}\right| d x d s \\
\leq & C \int_{0}^{t} \int_{0}^{1}\left[(1+\theta)\left|u_{x} \phi\right|+(1+\theta)^{q}\left|\theta_{t} \phi\right|\right] d x d s \\
\leq & \varepsilon \int_{0}^{t} \int_{0}^{1}(1+\theta)^{q+3} \theta_{t}^{2} d x d s+C \int_{0}^{t} \int_{0}^{1}(1+\theta)^{q-3} \phi^{2} d x d s \\
& +C\left(\int_{0}^{t} \int_{0}^{1}(1+\theta)^{2} u_{x}^{2} d x d s\right)^{\frac{1}{2}}\left(\int_{0}^{t} \int_{0}^{1} \phi^{2} d x d s\right)^{\frac{1}{2}} \\
\leq & \varepsilon X+C+C A^{q_{7}+\beta}+C\left(C+\varepsilon X+C A^{q_{3}}+C Z^{\frac{2}{3}}\right)^{\frac{1}{2}}\left(C+C A^{\beta}\right)^{\frac{1}{2}} \\
\leq & \varepsilon X+C+C A^{q_{7}+\beta}+\left(C+C A^{\frac{q_{3}}{2}}+C Z^{\frac{1}{3}}+\varepsilon X^{\frac{1}{2}}\right)\left(C+C A^{\frac{\beta}{2}}\right) \\
\leq & \varepsilon X+C+C A^{q_{7}+\beta}+C A^{\frac{q_{3}}{2}}+C Z^{\frac{1}{3}}+C X^{\frac{1}{2}}+C A^{\frac{\beta}{2}}+C A^{\frac{q_{3}+\beta}{2}} \\
& +C A^{\frac{\beta}{2}} Z^{\frac{1}{3}}+C A^{\frac{\beta}{2}} X^{\frac{1}{2}} \\
\leq & \varepsilon X+C+C A^{q_{7}+\beta}+C A^{\frac{q_{3}+\beta}{2}}+C Z^{\frac{1}{3}}+C A^{\frac{\beta}{2}} Z^{\frac{1}{3}} \\
\leq & \varepsilon(X+Y)+C+C Z^{\delta_{3}},
\end{aligned}
$$

where $\delta_{3}=\max \left(\frac{1}{3}, \frac{2 q+6}{3\left(2 q+6-\frac{\beta}{2}\right)}\right)$. Here in order to make the inequality (2.57) hold and $0<\delta_{3}<1$, we must require

$$
\left\{\begin{array}{l}
0 \leq q_{7}+\beta<2 q+6 \\
0 \leq \frac{q_{3}+\beta}{2}<2 q+6 \\
0 \leq \frac{\beta}{2}<2 q+6 \\
0 \leq \frac{2 q+6}{3\left(2 q+6-\frac{\beta}{2}\right)}<1
\end{array}\right.
$$

which implies

$$
\left\{\begin{array}{l}
0 \leq q_{7}+\beta<2 q+6, \\
0 \leq q_{3}+\beta<4 q+12, \\
0 \leq \beta<\frac{8}{3}(q+3) .
\end{array}\right.
$$

In a word, for $2 \leq q<3$ and $0 \leq \beta<2 q+6$ or $q \geq 3$ and $0 \leq \beta<q+9$, we can prove that (2.51), (2.55)-(2.58) hold, i.e.,

$$
0<\delta^{\prime}, \delta_{1}, \delta_{2}, \delta_{3}<1 .
$$

Combining (2.42)-(2.57) and taking $\varepsilon$ small, we obtain (2.41). Here if $2 \leq q<3$, then we may take

$$
\delta=\max \left(\frac{2}{3}, \frac{3}{4}, \delta^{\prime}, \delta_{1}, \delta_{2}, \delta_{3}\right)
$$

If $3 \leq q<4$, then we choose

$$
\delta=\max \left(\frac{2}{3}, \frac{3(q+3)}{2(2 q+5)}, \delta^{\prime}, \delta_{1}, \delta_{2}, \delta_{3}\right)
$$


and if $q \geq 4$, then we may pick

$$
\delta=\max \left(\frac{2}{3}, \frac{3(q+3)}{4(q+9)}, \delta^{\prime}, \delta_{1}, \delta_{2}, \delta_{3}\right) .
$$

The proof is now complete.

LEMma 2.8. If $2 \leq q<3$ and $0 \leq \beta<2 q+6$ or $q \geq 3$ and $0 \leq \beta<q+9$, i.e., $(q, \beta) \in E$, then for any $t \in[0, T]$,

$$
\begin{gathered}
\left\|u_{x}, u_{x x}, u_{t}, \theta_{x}, \theta_{x x}, \theta_{t}, z_{x}, z_{x x}, z_{t}\right\|^{2}+\int_{0}^{t}\left\|u_{x t}, \theta_{x t}, z_{x t}\right\|^{2} d s \leq C, \\
\left|u_{x}\right|^{(0)},|u|^{(0)},|\theta|^{(0)} \leq C, \\
\theta(x, t) \geq C, \quad \forall(x, t) \in \overline{Q_{T}} .
\end{gathered}
$$

Proof. Differentiating (1.2) with respect to $t$, multiplying it by $u_{t}$ and integrating it with respect to $x$, we have

$$
\frac{d}{d t} \int_{0}^{1} \frac{1}{2} u_{t}^{2} d x+\int_{0}^{1} \frac{\mu}{v} u_{x t}^{2} d x=\int_{0}^{1}\left(p_{t} u_{x t}+\frac{\mu}{v^{2}} u_{x}^{2} u_{x t}\right) d x .
$$

Since $p_{t}=\left(\frac{R}{v}+\frac{4}{3} a \theta^{3}\right) \theta_{t}-\frac{R}{v^{2}} \theta u_{x}$, for $\delta>\frac{3}{4}$, we get

$$
\begin{aligned}
\left\|u_{t}(t)\right\|^{2}+\int_{0}^{t}\left\|u_{x t}\right\|^{2} d s & \leq C \int_{0}^{t} \int_{0}^{1}\left(1+\theta^{6}\right) \theta_{t}^{2} d x d s+C\left|u_{x}^{2}\right|^{(0)} \int_{0}^{t}\left(\|\theta\|^{2}+\left\|u_{x}\right\|^{2}\right) d s \\
& \leq C(1+A)^{q_{2}} X+C+C Z^{\frac{3}{4}} \\
& \leq C+C Z^{\delta\left(1+\frac{q_{2}}{2 q+6}\right)} .
\end{aligned}
$$

From Lemma 2.7, by squaring (1.2) and noting $p_{x}=\left(\frac{R}{v}+\frac{4}{3} a \theta^{3}\right) \theta_{x}-\frac{R}{v^{2}} \theta v_{x}$, it follows from (2.62) that for any $t \in[0, T]$,

$$
\begin{aligned}
\left\|u_{x x}\right\| & \leq C\left[1+\left\|u_{t}\right\|^{2}+\int_{0}^{1}\left(1+\theta^{6}\right) \theta_{x}^{2} d x+\left(\left|\theta^{2}\right|^{(0)}+\left|u_{x}\right|^{(0)}\right)\left\|v_{x}\right\|^{2}\right] \\
& \leq C+C Z^{\delta\left(1+\frac{q_{2}}{2 q+6}\right)}+C Z^{\frac{3}{4}}+C A^{2}+C(1+A)^{2 q_{2}} Y \\
& \leq C+C Z^{\delta\left(1+\frac{2 q_{2}}{2 q+6}\right)}
\end{aligned}
$$

whence

$$
Z \leq C+C Z^{\delta\left(1+\frac{2 q_{2}}{2 q+6}\right)} .
$$

If $2 \leq q<3,0 \leq \beta<2 q+6$, then $q_{0}=4-q, q_{1}=8-q, q_{2}=3-q, q_{3}=8-q, q_{4}=$ $7-2 q, q_{*}=\frac{12-q}{2}, q_{6}=\frac{12-q}{2}, q_{7}=0$. We can easily prove that when $2 \leq q<3$ and $0 \leq \beta<2 q+6$,

$$
0<\delta\left(1+\frac{2 q_{2}}{2 q+6}\right)<1
$$

which thus gives

$$
Z \leq C .
$$


If $q \geq 3$ and $0 \leq \beta<q+9$, we can also prove that

$$
0<\delta\left(1+\frac{2 q_{2}}{2 q+6}\right)<1
$$

and

$$
Z \leq C
$$

which also implies (2.63). Hence we conclude that $Z$ is bounded, and we can see from Lemma 2.7 and the definitions of $A, X, Y, Z$ that $\left\|u_{x}, u_{x x}, u_{t}, \theta_{x}\right\|,|u, \theta|^{(0)}$ and $\int_{0}^{t}\left\|u_{x t}, \theta_{t}\right\|^{2} d s$ are also bounded. For the boundedness of other quantities in (2.59)(2.61), whose proofs are very standard and so we omit them here, we can refer to the proofs of Lemmas 10-13 in [27].

The proof of Theorem 1.2, and hence of Theorem 1.1, is complete.

Acknowledgments. This paper was supported in part by the NNSF of China (No. 10871040 and No. 11031003).

\section{REFERENCES}

[1] S. N. Antontsev, A. V. Kazhikhov and V. N. Monakhov, Boundary Value Problems in Mechanics of Nonhomogeneous Fluids, Amsterdam, New York, 1990. MR.1035212 (91d:76018)

[2] G. Chen, Global solutions to the compressible Navier-Stokes equations for a reacting mixture, SIAM J. Math. Anal., 23(1992), 609-634. MR1158824 (93h:76060)

[3] D. Donatelli and K. Triosia, On the motion of a viscous compressible radiative-reacting gas, Comm. Math. Phys., 265 (2006), 463-491. MR2231679 (2007b:35263)

[4] B. Ducomet, Hydrodynamical models of gaseous stars, Reviews Math. Phys., 8(1996), 957-1000. MR 1415382(97i:85001)

[5] B. Ducomet, A model of thermal dissipation for a one-dimensional viscous reactive and radiative gas, Math. Meth. Appl. Sci., 22(1999), 1323-1349. MR1710987 (2000g:76094)

[6] B. Documet, Some stability results for reactive Navier-Stokes-Poisson systems, in Evolution equations: existence, regularity and singularities (Warsaw, 1998), 83-118, Polish Acad. Sci., Polish Scientific Publishers, Warsaw, 2000. MR.1773094 (2001g:76043)

[7] B. Ducomet, E. Feireisl, H. Petzeltov and I. Straškraba, Existence globale pour un fluide barotrope autogravitant, C. R. Acad. Sci. Paris, Série I, 332(2001), 627-632. MR1841897 (2002e:76050)

[8] B. Ducomet and E. Feireisl, On the dynamics of gaseous stars, Arch. Rational Mech. Anal., 174(2004), 221-266. MR2098107 (2005m:35223)

[9] B. Ducomet and E. Feireisl, The equations of magnetohydrodynamics: on the interaction between matter and radiation in the evolution of gaseous stars, Comm. Math. Phys., 266(2006), 595-626. MR.2238891(2007d:76229)

[10] B. Ducomet and A. Zlotnik, Stabilization for viscous compressible heat-conducting media equations with nonmonotone state functions, C. R. Acad. Sci. Paris, Ser. I 334(2002),119-124. MR1885092 (2002k:35259)

[11] B. Ducomet and A. Zlotnik, Stabilization for equations of one-dimensional viscous compressible heat-conducting media with nonmonotone equation of state, J. Differential Equations, 194(2003), 51-81. MR2001029 (2004f:35135)

[12] B. Ducomet and A. Zlotnik, Stabilization for 1D radiative and reactive viscous gas flows, C. R. Acad. Sci. Paris, Ser. I 338(2004), 127-132. MR2038281 (2004k:76097)

[13] B. Ducomet and A. Zlotnik, On the large-time behavior of 1D radiative and reactive viscous flows for higher-order kinetics, Nonlinear Analysis, 63(2005), 1011-1033. MR:2211579 (2007a:76100)

[14] B. Ducomet and A. Zlotnik, Lyapunov functional method for 1D radiative and reactive viscous gas dynamics, Arch. Rat. Mech. Anal., 177(2005), 185-229. MR2188048(2006k:76113) 
[15] A. V. Kazhikhov and V. V. Shelukhin, Unique global solution with respect to time of initial boundary value problems for one-dimensional equations of a viscous gas, J. Appl. Math. Mech., 41(1977), 273282. MR0468593 (57:8425)

[16] O. A. Ladyžhenskaja, V. A. Solonnikov and N. N. Ural'ceva, Linear and quasi-linear equations of parabolic type, Transl. Math. Monogr., Vol. 23, Amer. Math. Soc., Providence, RI, 1968. MR0241821 (39:3159a)

[17] D. Mihalas and B. Weibel-Mihalas, Foundations of radiation hydrodynamics, Oxford Univ. Press, New York, 1984. MR781346 (86h:85004)

[18] T. Nagasawa, On the outer pressure problem of the one-dimensional polytropic ideal gas, Japan J. Appl. Math., 5(1)(1988), 53-85. MR924744 (89c:76078)

[19] G. Pomraning, The equations of radiation hydrodynamics, Pergamon Press, 1973.

[20] Y. Qin, Nonlinear Parabolic-Hyperbolic Coupled Systems and Their Attractors, Vol. 184, Operator Theory, Advances in PDEs, Basel-Boston-Berlin, Birkhäuser, 2008. MR2442890 (2010b:35315)

[21] Y. Qin, G. Hu, L. Huang and Z. Ma, Remarks on global solutions to a 1D self-gravitating viscous radiative and reactive gas, J. Math. Anal Appl., to appear.

[22] Y. Qin, L. Huang, S. Deng, Z. Ma, X. Su and X. Yang, On the 1D viscous reactive and radiative gas with the one-order Arrhenius kinetics, Preprint.

[23] P. Secchi, On the motion of gaseous stars in the presence of radiation, Comm. PDEs., 15(1990), 185-204. MR 1032628 (91a:35159)

[24] S. F. Shandarin and Ya. B. Zel'dovich, The large-scale structure of the universe: turbulence, intermittency, structures in a self-gravitating medium, Rev. Modern Phys., 61(1989), 185-220. MR $989562(89 \mathrm{~m}: 85008)$

[25] A. Tani, On the first initial-boundary value problem for compressibloe viscous fluid motion, Publ. Res. Inst. Math. Sci., 13(1977), 193-253.

[26] A. Tani, On the free boundary value problem for compressible viscous fluid motion, J. Math. Kyoto Univ., 21(4)(1981), 839-859. MR637520 (83e:35131)

[27] M. Umehara and A. Tani, Global solutions to one-dimensional equations for a self-gravitating viscous radiative and reactive gas, J. Differential Equations, 234(2007), 439-463. MR2300663(2007k:35377)

[28] M. Umehara and A. Tani, Global solvability of the free-boundary problem for one-dimensional motion of a self-gravitating viscous radiative and reactive gas, Proc. Japan Acad., 84(A)(2008), 123-128. MR2450064 (2009h:76189)

[29] M. Umehara and A. Tani, Temporally global solution to the equations for a spherically symmetric viscous radiative and reactive gas over the rigid core, Anal. Appl., 6(2008), 183-211. MR2405156 (2009h:35322)

[30] F. Williams, Combustion Theory, Benjamin/Cummings, Menlo Park, 1985. 